\title{
SMART REAL TIME EMBEDDED ARDUINO BASED DATA ACQUISITION SYSTEM
}

\author{
Saraswati Teli ${ }^{1}$, Mani. $\mathbf{C}^{2}$ \\ ${ }^{1}$ Asst. Professor, E \& C Dept, Maratha Mandal College of Engg\& Technology, Belgaum, India \\ ${ }^{2}$ Research Scholar, E \& C Dept, Maratha Mandal College of Engg\& Technology, Belgaum, India
}

\begin{abstract}
In this paper, an effort has been made to design and develop a smart real time embedded Arduino based data logger for indoor and outdoor environment. The present work has been concentrated to environmental parameters such as temperature, humidity and solar insolation. The data logger is proposed to be developed with the use of Arduino Uno based on ATmega328. The Arduino Microcontroller board is used which has inbuilt ADC and other peripheral circuitry necessary for operation. The physical parameter is sensed by the sensors and is converted into analog signal. This analog signal is fed to the Arduino board $A D C$ pins which is then converted in to an equivalent digital quantity and is further processed in the microcontroller. The raw digital signal or processed signal out of microcontroller may be displayed on the LCD display or it's saved in a database or even at the same time this data is sent to computer through the USB serial port. The serial port data is then accessed and is imported in Microsoft excel for computation and graphical representation.
\end{abstract}

Key Words: Arduino Uno, ATmega328, DHT 11, Solar cell, LCD etc.

\section{INTRODUCTION}

A data logger or a data recorder is an electronic measuring device, $\log$ s the data over a period of time with a sensor, built in instrument or via external instruments. The data logger measurements may include: temperature and humidity of air, alternating and direct current and voltage, air pressure, room occupancy, intensity of light, temperature of water, level of water, water content in soil, dissolved oxygen, measurement of rain, motion of wind and it's path, pulse signals, leaf wetness etc.

Data logging systems are generally based on a computer or any processor that processes in digital. These are usually little in size, portable, powered by battery and also they are provided with a computer processor, sensors and memory required for the storage of data. These are widely used inside the building, outside the building and in submarine circumstances where the data is required and where there is a convenience of battery power is preferred, and also they can record data months together at a time, unattended. This embedded unit may be a single one and self-contained device with existing physical sensors that detect the available data that fit in the hand, or it may be a multiplechannel device provided with many external sensors. These systems connect with a computer and use the particular software to run the data logger to review and determine the collected data, while other data loggers use a nearby interfacing devices like handheld keyboard or LCD and can be used as self-contained devices.

The purpose of having data loggers is its capacity to spontaneously collecting data in 24 hours cycle continuously without a break. After activating, the data loggers are left not attending to measure and log data for the particular date and time. This permits exact and complete image of the atmospheric conditions which are being observed such as temperature of air, moisture content in the air, pressure and solar insolation and many more parameters. The price of data loggers is varying day by day as the science and technology is improving and it depends on the number of channels. As the number of channels reduce, the cost reduces. Data loggers with simple and single channel cost as minimum as $\$ 25$. The more complex data loggers may cost very high

\section{BLOCK DIAGRAM AND ITS WORKING METHDOLOGY}

Data loggers in present market are too costly making them not to be feasible for important but low cost systems. The data acquired may be important in such systems but the cost of acquisition shoots up the system cost making it an economic constraint for project developers. The present DAQ systems are costly, this project intends to develop a product which beats the present market cost of the DAQ with additional features such as database storage with real time plotting of collected data. This concept is implemented using microcontrollers and sensor circuits which are developed and are custom designed as per the physical data to be captured.

Usual 8051 microcontroller uses external ADC which is interfaced with it and converts the analog signal fed into digital signal which is then fed to microcontroller for processing. Here in this project Arduino Microcontroller board is used which has inbuilt ADC and other peripheral circuitry necessary for operation. The physical parameter is sensed by the sensors and is converted into analog signal. This analog signal is fed to the Arduino board ADC pins 
which is then converted in to an equivalent digital quantity and is further processed in the microcontroller. The raw digital signal or processed signal out of microcontroller may be displayed on the LCD display or it's saved in a database or even at the same time this data is sent to computer through the USB serial port. The serial port data is then accessed and is imported in MATLAB, Python or Excel for computation and graphical representation.

This developed product can be readily implemented in industry for logging of any physical quantity such as temperature, humidity or pressure etc. The project is even suitable for digital signal processing of a physical signal into computational software like MATLAB or Microsoft Excel or even PYTHON.

The block diagram is as shown below:

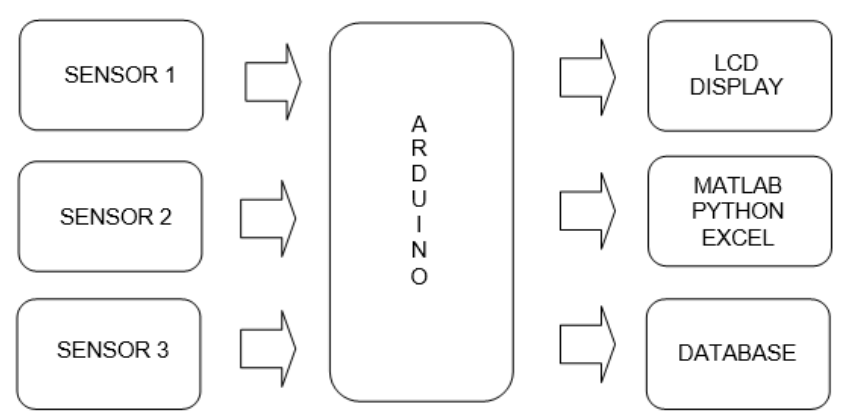

Fig 1: Block Diagram of Proposed Data Acquisition System

\section{SYSTEM COMPONENTS}

It consists of three components which are as follows:

1) Sensors

2) Arduino

3) LCD

\section{DESCRIPTION OF MODULES}

\section{1) Sensors}

Sensors are used to detect the physical data according to natural conditions. The DHT 11 sensor is used in this project is as follows:

1.1 DHT 11 :It has both temperature and humidity and is a complex digital measuring signal output. DHT 11 technology offers excellent long-term stability and high reliability. It is connected to 8-bit microcontroller. This sensor has a resistive component and also includes a sense of wet NTC temperature measuring instruments. It has high power, anti-interference capability, rapid response and quick implementation benefits. DHT 11 is very small in size and works on a very low power and it is capable to transmit a signal up-to-20 meter. In DHT 11, the digital output is directly proportional to the humidity and temperature and hence the name DHT 11 and it is measured by the sensor. Every DHT11 sensor is properly measured in the laboratory so that the internal OTP memory is used for the storage of calibration coefficient of DHT 11. The physical interfacing of sensor is realized through a 0.1 " pitch 3-pin connector: $+5 \mathrm{~V}$, GND and DATA. The sensor is powered by first two pins and the third pin is serial digital output signal of the sensor. Because of its small size and light weight, it is used for implementing data monitoring systems and small robots.

Fig 2: Pin out of DHT 11

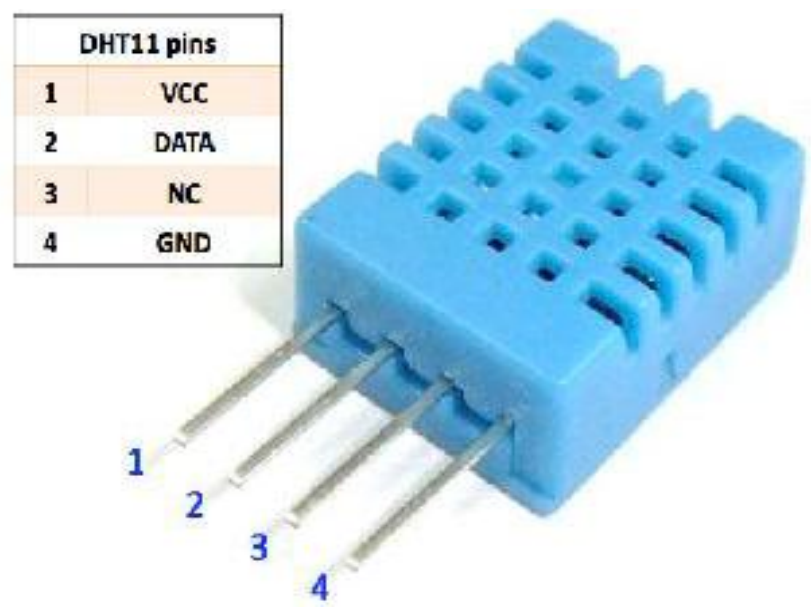

\subsection{Solar Cell for Solar Insolation Measurement}

A solar cell is a photovoltaic cell, which is a charged device that transforms light energy directly into electrical energy by a phenomenon called photovoltaic effect. Though the light source may be sunlight or an artificial light, these cells have been presented as photovoltaic cells. These are also used as a photodetector, detecting light or any other electromagnetic radiation.

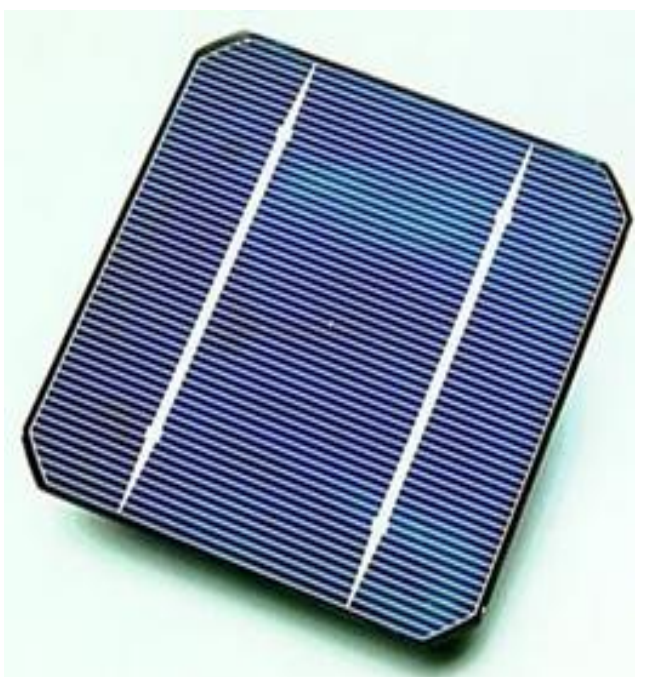

Fig 3: Solar Cell

Every day, we see that the sun delivers energy to the earth surface with free of charge. Any individual can use this freely available natural source. Thanks to a photovoltaic technology that converts the sun's energy directly into electricity. The Solar energy comes under the renewable energy sources that can be utilized effectively where there is huge electricity requirement throughout the world. The developments in solar technology have made the research endless which are required to be carried out in the present context of the world. 


\section{ARDUINO}

Arduino is electronics based open-source companyand easyto-use as hardware and software. This is highly useful for anyone to make effective projects. Arduino board accepts the inputs from various sensors that are connected to it by sensing environment and make changes in the surroundings by monitoring and controlling various devices. The Arduino environment has been designed in such a way that it's very handy and easy to understand especially for beginners who do not have the experience of using this tool for implementing low cost embedded projects. The objects built with Arduino respond to sound, control light, touch, and movement etc. The developed Arduino is used to produce an amazing variety of things such as games, musical instruments, robots, interactive furniture and also interactive clothing.

It is best suited for its hardware but its software that is set of instructions is also required to program hardware. The hardware and the software both include "Arduino." The physical world can be sensed and controlled by the combination of both hardware and software of Arduino that enable the user to create interactive projects. The Arduino software is freely available, cross-platform and also hardware is reasonable to buy. And also, there is an active and supportive Arduino community which can access worldwide through the Arduino council and the wiki.

\subsection{Arduino Software}

In Arduino software, using Arduino IDE, programs are generated on a computer. The code can be written and edited by IDE and it converts this code into a form of instructions so that it is understood by Arduino hardware. And also the instructions are transferred to the Arduino board by IDE. This process of transferring instructions is called uploading.

\subsection{Arduino Hardware}

The Arduino board is nothing but the code written is executed. This board can control and responds to electricity, so the components connected to the board can communicate with the real world. The components may be sensors, that convert physical quantity into electrical quantity and actuators that get electrical power from the board and are converted into some other form that changes the world. Sensors may be accelerometers, switches and ultrasound distance sensors. The actuators are mechanisms like LEDs, motors, speakers etc. The well-known Arduino boards consists of a USB connector, used to provide power and connectivity to the board for uploading the software. The figureshows a basic Arduino Uno board.

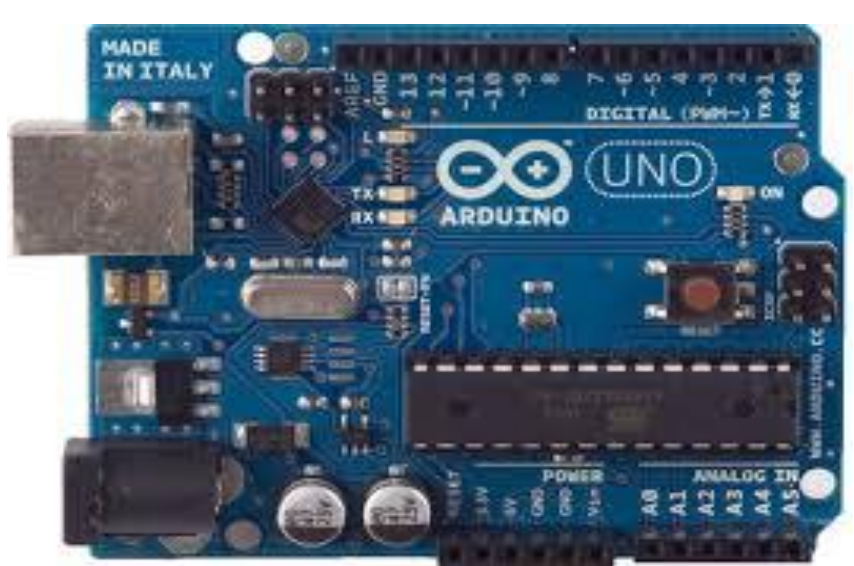

Fig 4: Basic Board of Arduino UNO.

Arduino Uno is a microcontroller board that consist of a total of 14 digital input/output pins, 6 analog input pins, an ICSP header, a power jack, a $16 \mathrm{MHz}$ oscillating crystal, a USB connection, and a reset button. Arduino Uno uses Atmega328 micro controller for powering up and contains everything that is essential to support the microcontroller. Arduino Uno can be interfaced to a laptop via USB cable. It doesn't use FTDI USB-to-serial driver chip. Generally, it uses the Atmega8u2 modified as a USB-to-serial converter. The Arduino board has power supply from a USB connection or it can be an external power supply where it comes either from a battery or from an AC-to-DC adapter.The Arduino board operates on $7 \mathrm{~V}$ to $20 \mathrm{~V}$ external supply. Suppose if the board is using supply voltage of less than $7 \mathrm{~V}$, then the $5 \mathrm{~V}$ pin may supply less than $5 \mathrm{~V}$ and there is a possibility that the board may get unstable. The Arduino board may get damaged or spoiled due to overheating if it uses higher external voltage of more than $12 \mathrm{~V}$. Thus the external supply of 7 to 12 volts is recommended range.

The ATmega328 has a flash memory of $32 \mathrm{~KB}$. This also has $2 \mathrm{~KB}$ of SRAM and $1 \mathrm{~KB}$ of EEPROM. The total 14 digital pins which are present on the board can be used either as an input or output.These digital pins function at 5 volts. Each input/output pin has direct current of $40 \mathrm{~mA}$ and these digital pins have an internal pull-up resistor of about 20-50k Ohms. Out of 14 digital I/O pins of Arduino, 6 pins provide PWM output (pin3, pin5, pin6 pin9, pin10and pin11). The DC current of $50 \mathrm{~mA}$ is for $3.3 \mathrm{~V}$ pin. The Arduino board consists of 6 analog inputs, each input provides 1024 different values or 10 bits of resolution. By default the analog input pins measure from 0 to 5 volts. The Arduino has a clock speed of about $16 \mathrm{MHz}$ and It can also be programmed with Arduino software.

\section{LCD}

A LCD is a wide, electronic display, which uses the light adjusting characteristics of liquid crystals. They do not exhibit light directly. Here, in our project we are going to use a solid monochromatic 20x4 alphanumeric LCD with Arduino. The 20x4 LCD means that the total of 20 characters in each row can be displayed. There are 4 such rows for $20 \times 4 \mathrm{LCD}$, thus 80 characters can be displayed on the LCD at any instance of time. 


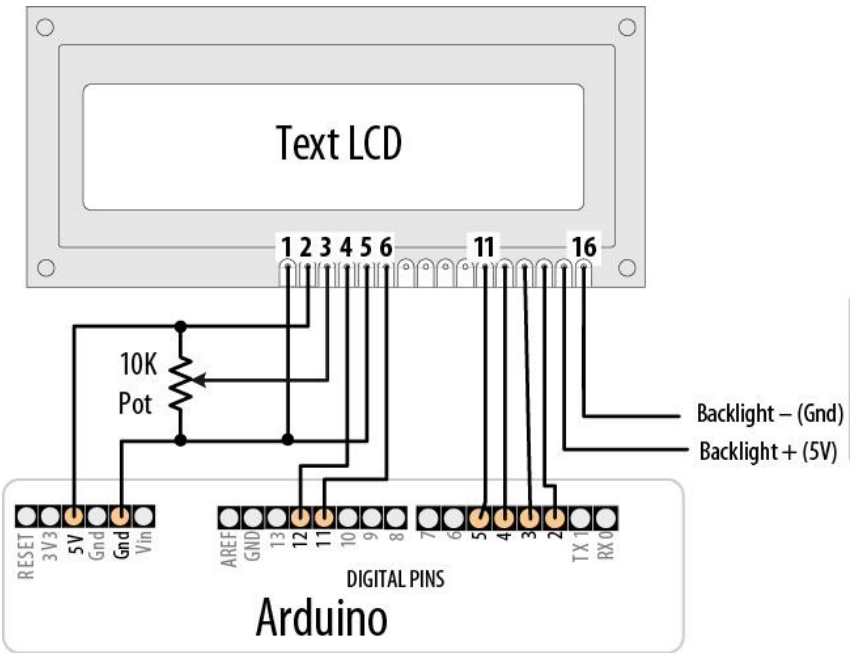

Fig 5: LCD to Arduino Uno Circuit

\section{OPERATION OF SYSTEM}

The idea behind this paper is to develop a smart real time embedded Arduino based data acquisition system. The present data loggers available in market are too costly and they can only monitor the data. Here the operation involves two steps data monitoring as well as data logging. This concept is implemented using Arduino microcontroller and sensor circuits which are developed and are custom designed as per the physical data to be captured. The data acquisition system is developed with additional features such as data base storage with real time plotting of collected data.

Initially simulation of the circuit is done using proteus simulation software to check whether the circuit designed for project will work out or not. After observing the simulation result in proteus, PCB design is made with it. Here we have used Arduino Uno tool for programming ATmega328. Then ATmega328 chip is removed and placed it on a PCB with required components. The Arduino microcontroller board is used which has inbuilt ADC and other peripheral circuitry necessary for operation. Two physical sensors named DHT 11 and solar cell are used. The DHT 11 is a digital humidity and temperature sensor which shows both digital temperature in degree celsius and humidity in percentage and it is connected to the ATmega328. The selenium or silicon solar cell is used as a physical sensor and it is connected to the analog pin of the ATmega328. The physical parameter is sensed by sensors and is converted into analog signal. This analog signal is fed to the Arduino board ADC pins which is then converted into an equivalent digital quantity and is further processed in the Arduino microcontroller. The solar insolation and the DHT 11 digital signal or processed signal out of the microcontroller is displayed on $20 * 4$ LCD display and also it is saved in a database. At the same time this data is sent to the computer through USB serial port. The serial port data is then accessed and is imported into the Microsoft Excel using PLX DAQ for computation and graphical representation. Hence we can see the graph of data logging of physical parameters with respect to time.

\section{RESULTS AND DISCUSSION}

Developed sensors are interfaced to microcontroller and are calibrated comparing with standard measuring instruments. The rig is tested for number of days, after calibration the parameters are plotted with respect to time of day. The variation of measured parameters is studied and suitable conclusions are drawn after few days of exhaustive field tests. These measured parameters are displayed in real time on the LCD of the data logger and as well as recorded/logged into excel sheet for further analysis.

\section{CONCLUSION}

Following are the conclusions drawn after fabrication and testing of the developed product from the proposed research work.

- Designed product is much cost effective compared to existing products.

- Product is industry ready for implementation.

- The system is fully automated and once it is setup no human intervention is required for monitoring. This will also reduce errors in measurements as because many times human intervention leads to errors.

- Designed system consumes very less power so this is also power efficient and can function autonomous with small dedicated battery for backup with grid connection even in power outages.

\section{FUTURE SCOPE}

- This system can be altered marginally to $\log$ and record other physical signals rather than temperature, humidity or pressure in requisite application.

- Product can be custom build to suit prerequisite accuracy and presision of measurement by upgrading the analog to digital converter rather than using inbuilt 10 bit converter embedded in microcontroller.

- Data aquired can be imported further into computational softwares like MATLAB, Python for mathematical computaions.

- Multiple channel data acquition can be implemented using collective number of sensors connected to analog or digital inputs respectively as per sensing parameter and sensor specifications.

\section{REFERENCES}

[1] Vandana Pandya, Deepali Shukla "GSM Modem Based Data Acquisition System" International Journal of Computational Engineering Research (ijceronline.com) Vol.2 Issue. 5 September 2012.

[2] Ms. VaishaliDhawale, Prof. S.M. Turkane "Data Acquisition System (DAS) Based on ARM Embedded Web Server" Ms. VaishaliDhawale et al Int. Journal of Engineering Research and Applications ISSN: 22489622, Vol. 4, Issue 1( Version 3), January 2014,pp.212-215

[3] Anuj Kumar, I. P. Singh, and S. K. Sud, "Design and Development of Multi-Channel Data Logger for Built Environment", International Multi Conference of 
Engineers and Computer Scientists 2010 Vol II, March 17-19, 2020, Hong Kong.

[4] A. Goswami, T. Bezboruah and K.C. Sarma "Design of An Embedded System For Monitoring and Controlling light and temperature" International Journal of Electronic Engineering Research Volume 1 Number 1 (2009) pp. 27-36.

[5] A.Rahali, M.Guerbaoui, A.Ed-dahhak, Y. El Afou1, A.Tannouche, A.Lachhab, B. Bouchikhi "Data acquisition Development and greenhouse control system based on GSM" International Journal of Engineering, Science and Technology Vol. 3, No. 8, 2011, pp. 297-306.

[6] Md. MoyeedAbrar, Rajendra R. Patil, "Multipoint Temperature Data Logger and Display on PC through Zigbee using PSoC.", International Journal of Advanced Research in Computer and Communication Engineering Vol. 2, Issue 9, September 2013.

[7] Mohammed Bilal "Real Time Attendance Logging With Multi Node Embedded System Connected Via Wifi" International Journal of Reconfigurable and Embedded Systems (IJRES) Vol. 1, No. 3, November 2012, pp. 103 107 ISSN: 2089-4864. 